\title{
Energy-Momentum Distribution for Magnetically Charged Black Hole Metric
}

\author{
M. Farasat Shamir ${ }^{1 *}$, Rida Ejaz ${ }^{1}$ and Mushtaq Ahmad ${ }^{2}$ \\ ${ }^{1}$ National University of Computer and Emerging Sciences, Lahore Campus, Pakistan. \\ ${ }^{2}$ National University of Computer and Emerging Sciences, Chiniot-Faisalabad Campus, Pakistan. \\ ${ }^{*}$ Corresponding author
}

\section{Article Info}

Keywords: Energy-momentum complex, Magnetically charged black hole metric.

2010 AMS: $83 C x x$

Received: 17 April 2019

Accepted: 6 April 2020

Available online: 24 April 2020

\begin{abstract}
This work investigates the well known localization problem of energy and momentum. The purpose of this paper is two fold. First, we compute Einstein, Landau-Lifshitz and Bergmann's energy-momentum complexes for static spherically symmetric magnetically charged regular black hole spacetime in general relativity. We observe strong coincidences among the results obtained form the three descriptions. These resembling results from different energy-momentum prescriptions may offer some basis to explain a exclusive quantity which supports Virabhadra's viewpoint. Secondly, the problem is discussed in modified gravity. In particular, we use generalized Landau-Lifshitz prescription for the determination of energy-momentum with reference to $f(R)$ theory of gravity. We explicitly compute the energy-momentum complex for the static spherically symmetric magnetically consistent regular black hole metric for a well-known choice of the $f(R)$ gravity models.
\end{abstract}

\section{Introduction}

The energy-momentum localization in curved spacetimes is one of the most important issues since the emergence of general relativity (GR). This problem has no proper solution till date. Several attempts have been carried out by researchers to overcome this issue, using different tools and hypothesis. A unique tensorial definition of energy and momentum has been a focus of many findings in the GR. Energy-momentum tensor $T_{a}^{b}$ is a second rank symmetric, localized and divergence-less quantity introduced in both, the special relativity and the classical mechanics. It gives the account of the energy and momentum matter source, and non-gravitational field sources. Given below equation defines the conservation law of energy and momentum

$$
T_{a, b}^{b}=0 .
$$

Unique definitions of energy and momentum exist in classical physics. However, ordinary derivatives transforms to covariant derivatives in GR. Thus, we get

$$
T_{a ; b}^{b}=\frac{1}{\sqrt{-g}}\left(\sqrt{-g} T_{a}^{b}\right)_{, b}-\Gamma_{a c}^{b} T_{b}^{c}=0 .
$$

This conservation law was formulated by Einstein [1]. Eq. (1.2) shows that $T_{a}^{b}$ does not satisfy Eq. (1.1) in the presence of gravitational field. The summation of these two terms (stress-energy tensor and a pseudo-tensor) remains divergence-less. The addition of a non-tensor quantity to justify the gravitational field energy was criticized by many researchers. Levi Civita argued on an alternate gravitational energy tensor. Penrose [2] introduced another concept of energy, known as quasi-local energy in order to find a feasible expression other than $t_{a}^{b}$ (pseudo-tensor). Pauli criticized Einstein's work on energy-momentum distribution but Einstien argued that his energy-momentum complex (EMC) gave reasonable outcomes for the energy and momentum of isolated systems which obey the conservation laws.

Email addresses and ORCID numbers: farasat.shamir@nu.edu.pk, https://orcid.org/0000-0002-3310-8806 (M. Farasat Shamir), ridaejaz362@gmail.com, (R. Ejaz),coolmushtaque@gmail.com, https://orcid.org/0000-0002-3775-926X (M. Ahmad) 
Later on, different prescriptions were suggested by many researchers including Landau-Lifshitz [3], Bergmann-Thomson [4], Tolman [5], Weinberg [6], Papapetrou [7], and Möller [8]. All of these works are coordinate dependent i.e one has to perform the computations in the quasi-cartesian coordinates except Möller's prescription which is coordinate free. Due to non-tensorial nature, these complexes are also called pseudo-tensors. Misner et al. [9] proved that spherical coordinate system can be used for the localization of energy. But later on, possibility of energy localization in any system was proved by Sarracino and Cooperstock [10] as they showed that the energy can be localized in any system, if it is localized in spherical systems. In 1990, Virabhadra claimed that energy-momentum complexes might provide intuitive conclusions just for the isolated systems total energy. Virabhadra and his co-authors [11] studied several spacetimes and found various energy distributions for such spacetimes. The issue of localization of energy-momentum in the GR gained a new viewpoint from the results elaborated by Xulu et al. [12]. Rosen [13] investigated the total energy of a closed homogenous isotropic universe using Einstein's EMC. Many efforts have been made to solve the problem of localization of energy and momentum [14]-[20]. Amir and Mirshekari [21] investigated energy-momentum distributions in static and non-static cosmic strings space-times using Einstein, Landau-Lifshitz, papapetrou, Bergmann-Thomson, Tolman, Mø̈ller, and Weinberg's prescriptions. They noted strong similarities between the findings. These results were thought to be the extension of Virabhadra's point of view that different energy-momentum prescriptions may provide some basis to explain an exclusive quantity. Xulu and his collaborators [12] investigated the matter source of black hole phantom metric with the help of Einstein's EMC. Sharif and Kanwal [22] evaluated energy-momentum distribution of Bell-Szekeres metric in the GR and teleparallel gravity with the help of Bergmann-Thomson, Einstein, Landau-Lifshitz, and Mø̈ller's prescriptions. Bergmann and Einstein's prescriptions for Bianchi type- $V$ spacetime in the GR and teleparallel theory of gravity were investigated by Salti and Aydogdu [23]. Bianchi type- $I I$ universe was investigated by Aydogdu using Landau-Lifshitz and Einstein EMC in the GR [24]. Banerjee and Sen [25] calculated the total energy density for Bianchi type-I universe using Einstein's EMC. Sharif and Fatima [26] computed the energy-momentum distribution of the Weyl metrics, singularity-free cosmological model and non-null Einstein-Maxwell solution using different prescriptions.

Modified theories of gravity have enthused many researchers lately due to the collective motivation imminent from high-energy physics, astrophysics, and cosmology. Among several alternatives to the Einstein's theory of gravity, theories which comprise high order scalar curvature invariants, and explicitly the class of $f(R)$ theories, enjoys a long history. In the last seven years or so, there has been a novel incentive for their study, leading to numerous fascinating results in this modified gravity. Furthermore, it has proven to be an effective and consistent to the GR and it continues to behave so. This definitely happens to be the reason to consider this theory for our present work Mainly, most of the work in the literature on the problem of energy momentum localization is with in the framework of the GR. In particular, the $f(R)$ gravity seems an interesting and straight forward modification for the discussion. Multämaki et al. [27] were the pioneers to generalize Landau-Lifshitz EMC in $f(R)$ theories of gravity. However, they restricted the generalization for those spacetimes having constant scaler curvature. Sharif and Shamir evaluated the energy density of plane symmetric solutions for some popular choices of $f(R)$ models. They also investigated the energy distribution of cosmic string spacetime [28]. Similar work can be found in [29]-[32].

Black holes gained much importance since Schwarzschild era. Xulu computed the energy distribution of Melvins magnetic universe and a charged dilaton black hole [12]. Gao and Zhang [33] investigated the properties of a phantom black hole metric. The accretion process of phantom fluid onto a black hole was studied by Babichev et al. [34]. Fabris and Bronnikov [35] obtained some interesting results on investigating the physics of neutral phantom black holes. Ding et al. [36] studied the influence of phantom fields on strong gravitational lensing.

In this paper, we interest to investigate some EMCs for static spherically symmetric magnetically charged regular black hole metric. For this purpose, we choose Landau-Lifshitz, Einstein, and Bergmann's prescriptions. We also investigate the energy and momenta for generalized Landau-Lifshitz EMC in $f(R)$ theory. The sequence of this manuscript is given as: Section 2 gives a brief discussion on the static spherically symmetric magnetically charged regular black hole metric. Section 3 is devoted to discuss different EMCs. In particular Einstein, Landau-Lifshitz and Bergmann's EMCs have been discussed for the magnetically charged regular black hole metric in detail. Section 4 gives the generalized expressions for Landau-Lifshitz in context of $f(R)$ gravity. Specifically, we calculate the components of energy and momentum. The last section is devoted to the comments and conclusive remarks.

\section{Magnetically charged regular black hole metric}

We use here the static, spherically symmetric magnetically charged regular black hole metric [37],

$$
d s^{2}=w(r) d t^{2}-\frac{d r^{2}}{w(r)}-r^{2} d \Omega^{2},
$$

where metric function $w(r)$ is given

$$
w(r)=1-\frac{2 m(r)}{r}
$$

with $m(r)$ is the mass function given by

$$
\begin{aligned}
m(r)= & \frac{q^{\frac{3}{2}}}{16 \sqrt[4]{\gamma}}\left[\ln \frac{2 \sqrt{\gamma} q-2 \sqrt[4]{\gamma} \sqrt{q} r+r^{2}}{2 \sqrt{\gamma} q+2 \sqrt[4]{\gamma} \sqrt{q} r+r^{2}}+2 \arctan \left(1+\frac{r}{\sqrt[4]{\gamma} \sqrt{q}}\right)\right. \\
& \left.-2 \arctan \left(1-\frac{r}{\sqrt[4]{\gamma} \sqrt{q}}\right)\right]
\end{aligned}
$$

where $q$ gives magnetic charge. To workout a black hole solution being consistent at $r=0$, the parameter $\gamma$ has to assume the value [37]

$$
\gamma=\left(\frac{\pi q^{\frac{3}{2}}}{8 M}\right)^{4}
$$


Thus the metric function $w(r)$ turns out to be

$$
\begin{aligned}
w(r)= & 1+\frac{M}{\pi r}\left[\ln \frac{32 M^{2} r^{2}+8 \pi M q^{2} r+\pi^{2} q^{4}}{32 M^{2} r^{2}-8 \pi M q^{2} r+\pi^{2} q^{4}}-2 \arctan \left(\frac{8 M r}{\pi q^{2}}+1\right)\right. \\
& \left.+2 \arctan \left(1-\frac{8 M r}{\pi q^{2}}\right)\right] .
\end{aligned}
$$

Here $M$ is the mass parameter. Ding and collaborators. [36] explored that for $M=0$, the metric provides the Ellis wormhole geometries. For the computation of components of energy and momentum, the line element (2.1)should be transformed from spherical polar coordinates $(t, r$, $\theta, \phi)$ to quasi-cartesian coordinates i.e. $(t, x, y, z)$ applying the following transformations

$$
\begin{aligned}
& x=r \cos \phi \sin \theta, \\
& y=r \sin \phi \sin \theta, \\
& z=r \cos \theta .
\end{aligned}
$$

The line element just transformed reads

$$
\begin{aligned}
d s^{2}= & w(r) d t^{2}-\left(d x^{2}+d y^{2}+d z^{2}\right)-\frac{1}{w(r)}\left(\frac{x d x+y d y+z d z}{r}\right)^{2} \\
& +\left(\frac{x d x+y d y+z d z}{r}\right)^{2}
\end{aligned}
$$

The corresponding determinant $g$ of the tensor $g_{i k}$ gives

$$
g=-1
$$

We obtain seven non-zero independent contra-variant components of the symmetric metric tensor.

$$
\begin{aligned}
g^{00} & =\frac{1}{w}, \\
g^{11} & =\frac{x^{2}(1-w)-r^{2}}{r^{2}}, \\
g^{22} & =\frac{y^{2}(1-w)-r^{2}}{r^{2}}, \\
g^{33} & =\frac{z^{2}(1-w)-r^{2}}{r^{2}}, \\
g^{12} & =\frac{(1-w) x y}{r^{2}}, \\
g^{23} & =\frac{(1-w) y z}{r^{2}}, \\
g^{31} & =\frac{(1-w) x z}{r^{2}} .
\end{aligned}
$$

\section{Energy-momentum prescriptions in the GR}

Here, we discuss the three different EMCs in the GR. In particular we discuss Einstein, Landau-Lifshitz and Bergmann's EMCs.

\subsection{Einstein energy-momentum prescription}

The Einstein EMC is given as[38]

$$
\Theta_{a}^{b}=\frac{1}{16 \pi} h_{a, c}^{b c}
$$

where

$$
h^{b c}{ }_{a}=-h^{c b}{ }_{a}=\frac{g_{a d}}{\sqrt{-g}}\left[-g\left(g^{b d} g^{c e}-g^{c d} g^{b e}\right)\right]_{, e} .
$$

The energy, momentum components are denoted by $\Theta_{0}^{0}$ and $\Theta_{i}^{0}$ respectively. $\Theta_{a}^{b}$ satisfies the covarient local Einstein's conservation laws

$$
\frac{\partial \Theta_{a}^{b}}{\partial x^{b}}=0
$$

The momentum four-vector (or the components of energy-momentum) is expressed as

$$
P_{a}=\iiint \Theta_{a}^{0} d x^{1} d x^{2} d x^{3} .
$$


$P_{i}$ with $(i=1,2,3)$ provides the components of momentum and the energy the energy is represented by $P_{0}$. Energy of physical system when the integration is taken over the hypersurface element by considering constant $t$ is

$$
E=\iiint \Theta_{0}^{0} d x^{1} d x^{2} d x^{3} .
$$

All the calculations are restricted to be carried out in quasi-cartesian coordinates. Applying Gauss's divergence theorem on the Eq. (3.1)leads to

$$
P_{a}=\frac{1}{16 \pi} \iint h^{0 b}{ }_{a} \mu_{b} d S
$$

where $\mu_{b}=\frac{x_{i}}{r}$ is the normal unit vector directed outward over an infinitesimal surface element $d S$. For the purpose to attain energy, we acquire three independent components of $h_{a}^{b c}$

$$
\begin{aligned}
h_{0}^{01} & =\frac{2 x(1-w)}{r^{2}}, \\
h^{02} & =\frac{2 y(1-w)}{r^{2}}, \\
h_{0}^{03} & =\frac{2 z(1-w)}{r^{2}} .
\end{aligned}
$$

Using the Eq. (3.3) in Eq. (3.2), the energy distribution is obtained as

$$
P_{E}(r)=\frac{r(1-w)}{4}
$$

where $P_{E}(r)$ expresses the total energy (gravitational field plus matter) within radius $r$. Likewise, $P_{i}$ gives the total momentum due to both gravitational field and matter. It is to be noted that

$$
\begin{aligned}
& h_{1}^{01}=h_{2}^{01}=h_{3}^{01}=0, \\
& h_{1}^{02}=h_{2}^{02}=h_{3}^{02}=0, \\
& h_{1}^{03}=h_{2}^{03}=h_{3}^{03}=0,
\end{aligned}
$$

suggesting that $P_{x}=P_{y}=P_{z}=0$. It is worthwhile to note that our results agree with [12] when we take $q=0$.

\subsection{Landau-Lifshitz energy-momentum prescription}

The EMC of Landau-Lifshitz is [38]

$$
L^{a b}=(-g)\left(T^{a b}+t^{a b}\right)=\frac{1}{16 \pi} \chi^{a b c d}{ }_{, c d},
$$

where $\chi^{a b c d}$ is defined as

$$
\chi^{a b c d}=-g\left(g^{a b} g^{c d}-g^{a c} g^{b d}\right) .
$$

The components of energy and momentum are expressed by $L^{00}$ and $L^{0 i}$ respectively.

$$
\frac{\partial L^{a b}}{\partial x^{b}}=0
$$

Eq. (3.4) further gives the conservation law for the quantity

$$
P^{a}=\iint(-g)\left(T^{a b}+t^{a b}\right) \mu_{b} d S .
$$

The quantities $t^{a b}$ vanish in the quasi-cartesian coordinates when there is no gravitational field, and the above relation takes the form

$$
P^{a}=\iint(-g) T^{a b} \mu_{b} d S
$$

which represents the four-momentum of the physical system. It gives the total four-momentum of the matter plus gravitational field. Thus, $t^{a b}$ is refereed as the energy-momentum pseudo-tensor of the gravitational field. The energy-momentum components are described as a three-dimensional integral space, given as

$$
P^{a}=\iiint 七^{a 0} d x^{1} d x^{2} d x^{3}
$$

Here also using Gauss's Theorem in Eq. (3.5), we get

$$
P^{a}=\frac{1}{16 \pi} \iint \chi_{, d}^{a 0 b d} \mu_{b} d S
$$


After some lengthy calculations the following independent components of $\chi^{a b c d}$ turn out to be

$$
\begin{aligned}
\chi^{0011} & =\frac{r^{2}+x^{2}(w-1)}{w r^{2}}, \\
\chi^{0022} & =\frac{r^{2}+y^{2}(w-1)}{w r^{2}}, \\
\chi^{0033} & =\frac{r^{2}+z^{2}(w-1)}{w r^{2}}, \\
\chi^{0012} & =\frac{-(w-1)}{x} y w r^{2}, \\
\chi^{0023} & =\frac{-(w-1)}{y} z w r^{2}, \\
\chi^{0031} & =\frac{-(w-1)}{x} z w r^{2} .
\end{aligned}
$$

Using Eq. the (3.7) in Eq. (3.6), we obtain

$$
P_{L}(r)=\frac{(1-w) r}{4 w}
$$

where $P_{L}(r)$ gives the total energy within radius $r$. Here we also get all the other components zero as expected from the geometry of a static metric.

\subsection{Bergmann energy-momentun prescription}

The Bergmann's EMC is given as[4]

$$
B^{a b}=\frac{1}{16 \pi} \beta_{, c}^{a b c}
$$

where $\beta^{a b c}$ is given as

$$
\beta^{a b c}=g^{a d} V_{d}^{b c}
$$

and $V_{d}^{b c}$ is defined as

$$
V_{d}^{b c}=-V_{d}^{c b}=\frac{g_{d e}}{\sqrt{-g}}\left[-g\left(g^{b e} g^{c f}-g^{c e} g^{b f}\right)\right]_{, f} .
$$

Here $B^{a b}$ approves the covariant local laws of conservation

$$
\frac{\partial B^{a b}}{\partial x^{b}}=0
$$

The energy-momentum components are expressed as

$$
P^{a}=\iiint B^{a 0} d x^{1} d x^{2} d x^{3}
$$

Applying the Gauss's theorem in the Eq. (3.8), we obtain

$$
P^{a}=\frac{1}{16 \pi} \iint \beta^{a 0 b} \mu_{b} d S
$$

Now, to obtain the energy distribution, we get just three components of $B^{a b c}$

$$
\begin{aligned}
B^{001} & =\frac{2 x(1-w)}{w r^{2}} \\
B^{002} & =\frac{2 y(1-w)}{w r^{2}} \\
B^{003} & =\frac{2 z(1-w)}{w r^{2}}
\end{aligned}
$$

Using Eqs. (3.10) in the Eq. (3.9), the energy distribution is obtained as

$$
P_{B}(r)=\frac{r(1-w)}{4 w}
$$

All the other components of $B^{a b c}$ are vanished resulting in zero momentum. It is worthwhile to mention here that the energy-momentum distribution for magnetically charged regular black hole metric in both Landau-Lifshitz and Bergmann prescription is same. Now, we investigate the energy-momentum distribution in modified $f(R)$ gravity. 


\section{Energy-momentum distribution in $f(R)$ theory of gravity}

Exploring the localization problem in alternative theories of gravity might be an intresting task. Researchers have worked on this localization issue in the teleparallel theory of gravity [39]-[41] expecting a positive solution of this complex problem. The energy-momentum distribution for particular space-times has been computed using different prescriptions by Sharif and Jamil [42]. No general conclusion was deduced form the results though they were consistent in some cases. Because of the cosmologically vibrant $f(R)$ model, the $f(R)$ theory has proven to be very attractive in the recent years. The generalization of Landau-Lifshitz EMC has been studied in the framework of this modified gravity [27].

\subsection{Generalized Landau-Lifshitz energy-momentum complex}

The $f(R)$ gravity is a generalised form of the GR. The $f(R)$ gravity is equipped with the following field equations

$$
F(R) R_{\mu \nu}-\frac{1}{2} f(R) g_{\mu \nu}-\nabla_{\mu} \nabla_{\nu} F(R)+g_{\mu \nu} \square F(R)=8 \pi G T_{\mu \nu}^{m},
$$

where $F(R)$ is the differentiation of $f(R)$ with respect to $R$. $T_{\mu \nu}^{m}$ defines the standard minimally coupled stress-energy tensor. The generalized Landau-Lifshitz EMC is given by [27]

$$
T^{\mu v}=F\left(R_{0}\right) \tau_{L L}^{\mu v}+\frac{1}{6 k^{2}}\left(F\left(R_{0}\right) R_{0}-f\left(R_{0}\right)\right) \partial_{\lambda}\left(g^{\mu v} x^{\lambda}-g^{\mu \lambda} x^{v}\right) .
$$

where $\tau_{\mu \nu}^{L L}$ represents the Landau-Lifshitz EMC worked out in the GR and $\kappa$ is the coupling constant. This is a generic expression validating any $f(R)$ theory of gravity when the scaler curvature of the chosen metric happens to be constant. The 00-component becomes

$$
T^{00}=F\left(R_{0}\right) \tau_{L L}^{00}+\frac{1}{6 k^{2}}\left(F\left(R_{0}\right) R_{0}-f\left(R_{0}\right)\right) \partial_{\lambda}\left(g^{00} x^{\lambda}-g^{0 \lambda} x^{0}\right),
$$

where $\tau_{L L}^{00}$ (the Landau-Lifshitz EMC) may also be computed the summation of energy-momentum and energy-momentum pseudo tensor as

$$
\tau_{L L}^{00}=(-g)\left(T^{00}+t_{L L}^{00}\right)
$$

where $t_{L L}^{00}$ is given by

$$
\begin{aligned}
t_{L L}^{00} & =\frac{1}{2 k}\left[\left(2 \Gamma_{\alpha \beta}^{\gamma} \Gamma_{\gamma \delta}^{\delta}-\Gamma_{\alpha \delta}^{\gamma} \Gamma_{\beta \gamma}^{\delta}-\Gamma_{\alpha \gamma}^{\gamma} \Gamma_{\beta \delta}^{\delta}\right)\left(g^{\mu \alpha} g^{v \beta}-g^{\mu v} g^{\alpha \beta}\right)\right. \\
& +\left(g^{\mu \alpha} g^{\beta \gamma}\right)\left(\Gamma_{\alpha \delta}^{v} \Gamma_{\beta \gamma}^{\delta}+\Gamma_{\beta \gamma}^{v} \Gamma_{\alpha \delta}^{\delta}-\Gamma_{\gamma \delta}^{v} \Gamma_{\alpha \beta}^{\delta}-\Gamma_{\alpha \beta}^{v} \Gamma_{\gamma \delta}^{\delta}\right) \\
& +\left(g^{v \alpha} g^{\beta \gamma}\right)\left(\Gamma_{\alpha \delta}^{\mu} \Gamma_{\beta \gamma}^{\delta}+\Gamma_{\beta \gamma}^{\mu} \Gamma_{\alpha \delta}^{\delta}-\Gamma_{\gamma \delta}^{\mu} \Gamma_{\alpha \beta}^{\delta}-\Gamma_{\alpha \beta}^{\mu} \Gamma_{\gamma \delta}^{\delta}\right) \\
& \left.+\left(g^{\alpha \beta} g^{\gamma \delta}\right)\left(\Gamma_{\alpha \gamma}^{\mu} \Gamma_{\beta \delta}^{v}-\Gamma_{\alpha \beta}^{\mu} \Gamma_{\gamma \delta}^{v}\right)\right] .
\end{aligned}
$$

It may be noted we need here the cartesian coordinates to apply the general formula in the Eq. (4.2). For convenience and without any loss of generality, we discuss the energy-momentum distribution of magnetically charged regular black hole metric in $f(R)$ gravity using polar coordinates.

\subsection{Energy distribution of magnetically charged regular black hole metric}

It is mentioned here that the value of scaler curvature for the metric (2.1) is

$$
R=\frac{2}{r}\left(\frac{d^{2} m}{d r^{2}}-\frac{d m}{d r}\right)
$$

On putting the value of $m(r)$ the Ricci scaler comes out to be constant i.e $R_{0}=0$. Hence, the static spherically symmetric magnetically charged regular black hole metric is an exact solution of any $f(R)$ theory, that satisfies the constant curvature condition. Now, as the Ricci scaler $R$ remains constant, we may find the energy density for this $f(R)$ model by implementing the generalized Landau-Lifshitz EMC. Using the Eq. (4.2) comes out with the following 00-component

$$
\tau^{00}=F\left(R_{0}\right) \tau_{L L}^{00}+\frac{1}{2 \kappa}\left(F\left(R_{0}\right) R_{0}\right)-f\left(R_{0}\right)(r F(R)+3 f(R)) .
$$

Using Eq. (4.3) and after some manipulations, we get

$$
\begin{aligned}
\tau_{L L}^{00} & =\frac{1}{2 \kappa^{2}}\left[\left(\frac{-3}{2} \frac{w^{\prime}(r)^{2}}{w(r)^{2}}-r w^{\prime}(r)-r w^{\prime}(r) \sin ^{2} \theta-\frac{7}{r^{2}}+2 w(r)-2\left(\frac{\cos ^{2} \theta}{\sin ^{2} \theta}\right)\right.\right. \\
& \left.+w(r) \sin ^{2} \theta+\frac{-3}{2} \frac{w^{\prime}(r)}{r w(r)}-\frac{2}{r} \frac{\cos \theta}{\sin \theta}\right)\left(1+\frac{1}{r^{2} w(r)}+\frac{1}{r^{2} w(r) \sin ^{2} \theta}\right) \\
& \left.+\left(\frac{1}{w(r)^{2}}\right)\left(\frac{w^{\prime}(r)^{2}}{2 w^{\prime}(r)}+\frac{2 w^{\prime}(r)}{r w(r)}\right)\right] .
\end{aligned}
$$


Substituting Eq. (4.5) in Eq. (4.4), it follows that

$$
\begin{aligned}
\tau^{00} & =\frac{1}{2 \kappa^{2}}\left[\left(\frac{-3}{2} \frac{w^{\prime}(r)^{2}}{w(r)^{2}}-r w^{\prime}(r)-r w^{\prime}(r) \sin ^{2} \theta-\frac{7}{r^{2}}+2 w(r)-2\left(\frac{\cos ^{2} \theta}{\sin ^{2} \theta}\right)\right.\right. \\
& \left.+w(r) \sin ^{2} \theta+\frac{-3}{2} \frac{w^{\prime}(r)}{r w(r)}-\frac{2}{r} \frac{\cos \theta}{\sin \theta}\right)\left(1+\frac{1}{r^{2} w(r)}+\frac{1}{r^{2} w(r) \sin ^{2} \theta}\right) \\
& \left.+\left(\frac{1}{w(r)^{2}}\right)\left(\frac{w^{\prime}(r)^{2}}{2 w^{\prime}(r)}+\frac{2 w^{\prime}(r)}{r w(r)}\right)\right] F\left(R_{0}\right) \\
& +\frac{1}{6 k^{2}}\left(F\left(R_{0}\right) R_{0}-f\left(R_{0}\right)\right)\left(\frac{-r w^{\prime}(r)}{w(r)^{2}}+\frac{3}{w(r)}\right) .
\end{aligned}
$$

Now we discuss an important case by choosing a $f(R)$ model. It is to be noted that we can not consider a model which becomes non-analytic at $R_{0}=0$. For example we can not choose $f(R)=R+\frac{c_{2}}{R}$. Thus the simplest and commonly used model is

$$
f(R)=R+c_{1} R^{2}
$$

For this model, the corresponding 00-component of the Landau-Lifshitz EMC, reads as

$$
\begin{aligned}
\tau^{00} & =\tau_{L L}^{00}(1)=\frac{1}{2 \kappa^{2}}\left[\left(\frac{-3}{2} \frac{w^{\prime}(r)^{2}}{w(r)^{2}}-r w^{\prime}(r)-r w^{\prime}(r) \sin ^{2} \theta-\frac{7}{r^{2}}+2 w(r)-2\left(\frac{\cos ^{2} \theta}{\sin ^{2} \theta}\right)\right.\right. \\
& \left.+w(r) \sin ^{2} \theta+\frac{-3}{2} \frac{w^{\prime}(r)}{r w(r)}-\frac{2}{r} \frac{\cos \theta}{\sin \theta}\right)\left(1+\frac{1}{r^{2} w(r)}+\frac{1}{r^{2} w(r) \sin ^{2} \theta}\right) \\
& \left.+\left(\frac{1}{w(r)^{2}}\right)\left(\frac{w^{\prime}(r)^{2}}{2 w^{\prime}(r)}+\frac{2 w^{\prime}(r)}{r w(r)}\right)\right] .
\end{aligned}
$$

\subsection{Momentum Of magnetically charged regular black hole metric}

We also calculate the momenta of the magnetically charged regular black hole metric by implementing the same technique and relations. $\tau^{0 i}$ represents the components of momentum. For momentum calculation, Eq. (4.1) becomes

$$
T^{0 i}=F\left(R_{0}\right) \tau_{L L}^{0 i}+\frac{1}{6 k^{2}}\left(F\left(R_{0}\right) R_{0}-f\left(R_{0}\right)\right) \partial_{\lambda}\left(g^{0 i} x^{\lambda}-g^{0 \lambda} x^{i}\right)
$$

Using Eq. (4.6), the simplified momentum components for $i=1,2,3$ are

$$
\begin{aligned}
\tau^{01}= & \frac{1}{2 \kappa^{2}}\left[\left(\frac{2 w(r) w^{\prime}(r)}{r}-\frac{2 w^{\prime}(r)}{r w(r)}+(2 w(r)+3) \cos ^{2} \theta-\frac{w^{\prime}(r)^{2}}{w(r)^{2}}-\frac{w^{\prime}(r)^{2}}{2}-\right.\right. \\
& \left.2 \frac{\cos ^{2} \theta}{\sin ^{2} \theta}\right)(-1)+\left(-1-\frac{1}{w(r)^{2}-\frac{1}{r^{2} w(r)}}-\frac{1}{r^{2} w(r) \sin ^{2} \theta}\right) \\
& \left(\frac{w(r) w^{\prime}(r)}{2 r}+r w(r) \sin \theta \cos \theta\right)+\left(-1+w(r)^{2}+\frac{w(r)}{r^{2}}+\frac{w(r)}{r^{2} \sin ^{2} \theta}\right) \\
& \left.\left(\frac{w^{\prime}(r)^{2}}{2 w(r)^{2}}-\frac{2 w^{\prime}(r)}{r w(r)}\right)\right], \\
& =\frac{1}{2 \kappa^{2}}\left[\left(\frac{-w^{\prime}(r)^{2}}{2}+\frac{2 w(r) w^{\prime}(r)}{r}-2 w(r)-2 f(r) \sin ^{2} \theta\right.\right. \\
& \left.-\frac{w^{\prime}(r)^{2}}{2 w(r)} \frac{2}{r} \frac{\cos \theta}{\sin \theta}-\cos ^{2} \theta-\frac{2 w^{\prime}(r)}{r w(r)}-2\left(\frac{\cos ^{2} \theta}{\sin ^{2} \theta}\right)\right)\left(\frac{-1}{r^{2} w(r)}\right) \\
& \left.+\left(-1-\frac{1}{w(r)^{2}}-\frac{1}{r^{2} w(r)}-\frac{1}{r^{2} w(r) \sin ^{2} \theta}\right)\left(\frac{-4 \sin ^{2} \theta \cos \theta}{r}\right)\right]
\end{aligned}
$$




$$
\begin{aligned}
\tau^{03}= & \frac{1}{2 \kappa^{2}}\left[\left(\frac{2 w(r) w^{\prime}(r)}{r}+\frac{2 w^{\prime}(r)}{r w(r)}-2 w(r)-3 w(r) \sin ^{2} \theta+4 \cos ^{2} \theta\right.\right. \\
- & \left.\frac{w^{\prime}(r)^{2}}{2}-\frac{w^{\prime}(r)^{2}}{2 w(r)^{2}}-\frac{2}{r^{2}}-\frac{2 \cos ^{2} \theta}{\sin ^{2} \theta}+\frac{w^{\prime}(r)}{2 w^{\prime}(r) r}-\frac{2 \cos \theta}{r \sin \theta}+\frac{w^{\prime}(r) \cos \theta}{2 w(r) \sin \theta}\right) \\
& \left(\frac{-1}{w(r) r^{2} \sin ^{2} \theta}\right)+\left(-1+\frac{1}{w(r)^{2}}-\frac{1}{w(r) r^{2}}-\frac{1}{w(r) r^{2} \sin ^{2} \theta}\right) \\
& \left(-w(r) \sin ^{2} \theta+\frac{2}{r^{2}}+\frac{2 \cos ^{2} \theta}{\sin ^{2} \theta}+\cos ^{2} \theta\right)+\left(\frac{-1}{r^{2} w(r) \sin ^{2} \theta}-\frac{w(r)}{r^{2} \sin ^{2} \theta}\right) \\
& \left.\left(\frac{3}{4} \frac{w^{\prime}(r)^{2}}{w(r)^{2}}-\frac{2 w^{\prime}(r)}{r w(r)}\right)\right]
\end{aligned}
$$

\section{Concluding remarks}

Many investigations have been put forward to address the issue of energy-momentum as it is an important conserved quantity. Unfortunately, there does not exist a general definition of energy and momentum in the GR. In this work, we focus to investigate the well-known problem of localization of energy-momentum with reference to the GR by using these three EMCs and also give some analysis under the modified gravity. In particular, we calculate the energy and momentum distributions for a static spherically symmetric magnetically charged regular black hole metric using Einstein, Bergmann-Thomson and Landau-Lifshitz EMCs. We conclude that energy turns to be well-defined and finite in these prescriptions for the black hole metric. It is worth noting that for $w=1$ the final results of all these three prescriptions gives constant energy equal to zero. It is to be noted that the unique results are obtained for Einstein, Bergmann-Thomson and Landau-Lifshitz energy-momentum prescriptions. Extension of Virbhadra's viewpoint [43] (different energy-momentum prescriptions may provide some basis to define a unique quantity) is supported by the coincidences observed in the results of these prescriptions. It is worthwhile to mention here that our results agree with [12] when we ignore the magnetic charge, i.e. $q=0$.

We have also worked on the energy and momentum distributions of the same metric in the context of modified gravity. For this purpose, we choose $f(R)$ theory of gravity and the Landau-Lifshitz energy-momentum prescription. Inspired by the recent interesting $f(R)$ gravity models, we generalize the results obtained for Landau-Lifshitz prescription. Here, we limit ourselves to investigate the Landau-Lifshitz EMC using the constant curvature assumption. The obtained energy and momentum components are well-defined for a space $r>0$. It would be an attractive task to get more generalized results by evaluating the Landau-Lifshitz EMC for non-constant Ricci scaler. Extending other EMCs in the context of $f(R)$ gravity as well in other modified theories of gravity would also be interesting.

For the comparative analysis of Energy-Momentum Distribution for Magnetically Charged Black Hole Metric, we have noted some worthy works in modified $f(\mathscr{R})$ theories of gravity in [29], [27], and [28] under some specific assumptions of different models and parameters. It has been noted that our work about Einstein, Landau-Lifshitz and Bergmann's energy-momentum complexes for static spherically symmetric magnetically charged regular black hole metric is consistent and similar in many aspects. However, a very few dissimilarities wherever they have appeared might be because of some different choices of the models and the corresponding parameters.

\section{References}

[1] C. Møller, The Theory of Relativity Oxford Univ. Press, London, (1958).

[2] R. Penrose, Quasi-local mass and angular momentum in general relativity, Proc. R. Soc. Lond. Ser. A Math. Phys. Eng. Sci., A381 (1982), $53-63$.

[3] L. D. Landau, E. M. Lifshitz, The Classical Theory of Fields, Pergamon Press, 1987.

[4] P. G. Bergmann, R. Thomson, Spin and angular momentum in general relativity, Phys. Rev., 89 (1953), 400.

[5] R. C. Tolman, On the use of the energy-momentum principle in general relativity, Phys. Rev. 35 (1930), 875.

[6] S. Weinberg, Gravitation and Cosmology: Principles and Applications of the General Theory of Relativity, John Wiley and Sons Inc., New York, 1972.

[7] A. Papapetrou, Einstein's theory of gravitation and flat space, Proceedings of the Royal Irish Academy. Section A, Mathematical and Physical Sciences, Royal Irish Academy, A52 1948, pp. 11-23.

[8] C. Møller, On the localization of the energy of a physical system in the general theory of relativity, Ann. Physics, 4 (1958), 347-371.

[9] C. W. Misner, K. S. Thorne, J. A. Wheeler, Gravitation, W. H. Freeman and Company, San Francisco, 1973.

[10] F. I. Cooperstock, R. S. Sarracino, The localisation of energy in general relativity, J. Phys. A: Math. Gen., 11 (1978), 877.

[11] A. Chamorro, K. S. Virbhadra, A radiating dyon solution, Pramana-J. Phys., 45 (1995), 181.

[12] P. K. Sahoo, K. L. Mahanta, D. Goit, A. K. Sinha, S. S. Xulu, U. R. Das, A. Prasad, and R. Prasad, Einstein energy-momentum complex for a phantom black hole metric, Chinese Physics Letters, 32(2) (2015), 020402.

[13] N. Rosen, The energy of the universe, Gen. Relativ. Gravit, 26 (1994), 319-321.

[14] S. S. Xulu, Energy distribution in Melvin's magnetic universe, Int. J. Modern Physics A, 15(30) (2000), 4849-4856.

[15] S. S. Xulu, Total energy of the Bianchi type I universes, Int. J. Theor. Phys., 39 (2000), 1153-1161.

[16] S. S. Xulu, Møller energy for the Kerr-Newman metric, Modern Phys. Lett. A, 15 (2000), 1511-1517.

[17] S. S. Xulu, Bergmann-Thomson energy-momentum complex for solutions more general than the Kerr-Schild class, Int. J. Theor. Phys., 46 (2007), 2915-2922.

[18] S. S. Xulu, Bergmann-Thomson energy of a charged rotating black hole, Found. Phys. Lett., 19 (2006), 603-609.

[19] A. K. Sinha, G. K.Pandey, A. K. Bhaskar, B. C. Rai, A. K. Jha, S. Kumar and S. S. Xulu, Effective gravitational mass of the Ayón-Beato and García metric, Modern Phys. Lett. A, A 30 (2015), 1550120.

[20] S. Aygün, I. Tarhan,Energy-momentum localization for Bianchi type-IV Universe in general relativity and teleparallel gravity, Pramana, 78 (2012), $531-548$.

[21] A. M. Abbassi, S. Mirshekari and A. H. Abbassi, Energy-momentum distribution in static and nonstatic cosmic string space-times, Phys. Rev., D 78 (2008), 064053

[22] M. Sharif, K. Nazir, Energy-momentum problem of Bell-Szekeres metric in general relativity and teleparallel gravity, Brazilian J. Phys., 38 (2008), $156-166$.

[23] O. Aydogdu, M. Salti, The momentum 4-vector in bulk viscous Bianchi type-V space-time, Czechoslovak J. Phys., 56 (2006), $789-798$.

[24] O. Aydogdu, Gravitational energy-momentum density in Bianchi type II space-times, Int. J. Modern Phys., D1504 (2006), $459-468$.

[25] N. Banerjee, S. Sen, Einstein pseudotensor and total energy of the universe, Pramana, 49 (1997), 609-615.

[26] M. Sharif, T. Fatima, Energy-momentum distribution: A crucial problem in general relativity, Int. J. Modern Phys., A20 (2005), 4309-4330. 
[27] T. Multamäki, A. Putaja, I. Vilja, E. C. Vagenas, Energy-momentum complexes in $f(R)$ theories of gravity, Classical Quantum Gravity, 25 (2008), 075017.

[28] M. Sharif, M. F. Shamir, Energy distribution in $f(R)$ gravity, Gen. Relativity Gravitation, 42 (2010), 1557-1569.

[29] M. Salt1, M. Korunur, İ. Açıkgöz, Gödel-type spacetimes in $f(R)$-gravity, Cent. Eur. J. Phys. 11.7 (2013), 961-967.

[30] M. Salti, O. Aydogdu, Energy in the Schwarzschild-de Sitter spacetime, Found. Phys. Lett., 19(3) (2006), 269-276.

[31] I-C. Yang, C-T. Yeh, R-R. Hsu, C-R. Lee, On the energy of a charged dilaton black hole, Internat. J. Modern Phys. A D 6(03) (1997), 349-356.

[32] I. Radinschi, The energy of a dyonic dilaton black hole, Acta Phys. Slov., 49 (1999), 789-794.

[33] C. J. Gao, S. N. Zhang, Phantom black holes, (2006), arXiv:hep-th/0604114.

[34] E. Babichev, V. Dokuchaev, Y. Eroshenko, Black hole mass decreasing due to phantom energy accretion, Phys. Rev. Lett., 93 (2004), 021102.

[35] K. A. Bronnikov, J. C. Fabris, Regular phantom black holes, Phys. Rev. Lett., 96 (2006), 251101.

[36] C. Ding, C. Liu, Y. Xiao, L. Jiang, R. G. Cai, Strong gravitational lensing in a black-hole spacetime dominated by dark energy, Phys. Rev. D, 88 (2013),

[37] M. S. Ma, Magnetically charged regular black hole in a model of nonlinear electrodynamics, Ann. Physics, 362 (2015), $529-537$.

[38] K. S. Virbhadra, Naked singularities and Seifert's conjecture, Phys. Rev., D60 (1999), 104041.

[39] G. G. Nashed, General spherically symmetric nonsingular black hole solutions in a teleparallel theory of gravitation, Phys. Rev., D66 (2002), 064015.

[40] T. Vargas, The energy of the universe in teleparallel gravity, Gen. Relativity Gravitation, 30 (2004), $1255-1264$.

[41] J. W. Maluf, J. F. da Rocha-Neto, T. M. L. Toribio, K. H. Castello-Branco,Energy and angular momentum of the gravitational field in the teleparallel geometry, Phys. Rev., D65 (2002), 124001.

[42] M. Sharif, M. J. Amir, Teleparallel versions of Friedmann and Lewis-Papapetrou spacetimes, Gen. Relativity Gravitation, 38 (2006), $1735-1745$.

[43] J. M. Aguirregabiria A. Chamorro, K. S. Virbhadra, Energy and angular momentum of charged rotating black holes, Gen. Relativity Gravitation, 28 (1996), 1393-1400. 